\title{
Simulation Studies for Predicting Surgical Outcomes in Breast Reconstructive Surgery
}

\author{
Celeste Williams $^{1}$, Ioannis A. Kakadaris ${ }^{1}$, K. Ravi-Chandar ${ }^{2}$, \\ Michael J. Miller ${ }^{3}$, and Charles W. Patrick ${ }^{3}$ \\ 1 Visual Computing Lab, Dept. of Computer Science \\ University of Houston, \\ 4800 Calhoun, Houston, TX \\ \{cbwilliams, ioannisk\}@uh.edu \\ http://www.vcl.uh.edu/〜ioannisk \\ ${ }^{2}$ Dept. of Aerospace Engineering \\ University of Texas at Austin, Austin, TX \\ kravi@mail . utexas .edu \\ 3 Dept. of Plastic Surgery \\ UT M.D. Anderson Cancer Center, \\ Houston, TX \\ $\{$ mmiller, cpatrick\}@mdacc.tmc.edu
}

\begin{abstract}
Currently, there is no reliable method to accurately predict the outcome of breast reconstruction for an individual patient prior to surgery. To address this limitation, we are developing a simulation system capable of rendering patient-specific predictions of the post-operative breast shape. Such a system for the planning of breast reconstructive surgery will greatly enhance patient prognosis. In this paper, we present our computer simulations representing the reconstructed breast using idealized and realistic geometric models.
\end{abstract}

\section{Introduction}

With the exception of skin cancer, breast cancer is the most common cancer diagnosis among women, and breast reconstruction is the third most frequently performed reconstructive surgery. After a mastectomy, breast volume can be replaced either by an implant or by the patient's own tissues (autologous tissues), such as in various tissue flaps. The method of reconstruction chosen depends on factors such as preference of the patient, patient's physical condition, and surgeon's expertise.

Implant-based reconstruction is generally easier to perform than reconstruction with autologous tissues and it requires less surgery. The implant is placed under the pectoralis major muscle and the muscle is stretched gradually with the aid of a tissue expander or adjustable implant that is injected with saline. For reconstruction with autologous tissue, breast volume is replaced with tissue from a donor site, usually the abdomen. Thus, the surgical procedure is more complicated. The most common procedure performed to acquire tissue is 
the transverse rectus abdominis myocutaneous (TRAM) flap. Two specific flap techniques commonly performed use the pedicle TRAM or the free TRAM [1. In these techniques, a wide ellipse of skin and fatty tissue is removed from the patient's abdomen and is either left attached to one of the two rectus muscles (pedicle TRAM), or completely removed along with a small plug of muscle (free TRAM). The flap is then shaped to form the breast mound.

Currently, there is no method to accurately predict the final breast shape and size in an individual patient after a surgical procedure. This is especially a challenge in reconstructive breast surgery where the goal is to achieve a natural appearance with symmetry. The current breast reconstructive procedure is performed based on the subjective judgment of the surgeon. A method, based on patient specific parameters and tissue mechanics, to determine the shape and size of the reconstructed breast prior to surgery will be very beneficial for both the patient and the surgeon.

In this paper, we describe our computer simulations capable of rendering predictions of the post-operative breast shape by using physical properties. The ultimate objective of this work is to develop the computational framework to integrate medical imaging and biomechanical data, in order to accurately predict changes in the three-dimensional breast shape resulting from any reconstructive procedure.

\section{Methods}

To perform simulations and predict the overall response of a reconstructed breast in the upright position and subjected to physical forces, numerical studies were performed in both 2D and 3D.

2D Numerical Modeling: Initial studies of simple 2D analysis were carried out using two approaches in order to gain intuition of the constraints and the initial conditions of the shape deformation problem [6]. In the first approach, we performed numerical simulations of a space curve with an applied normal force, which varied with height along the curve, in order to determine the shape and tension of the curve. Such simulations were equivalent to filling a surface bounded by the space curve with a liquid (e.g., a fluid-filled inextensible membrane). The free body diagram of such a curve, shown in Figure 1 with an arbitrary load, q, acting on the curve $(d s)$, yields the equilibrium equation,

$$
\frac{d \mathbf{T}(s)}{d s}+\mathbf{q}(s)=\mathbf{0}
$$

where $\mathbf{T}$ is the tension of the membrane curve and it is along the tangent $\mathbf{t}$.

If the load is expressed as,

$$
\mathbf{q}(s)=-\gamma\left(\frac{h}{2}-y(s)\right) \mathbf{k} \times \boldsymbol{t}(s)
$$

such that force varies with height or depth along the curve, $y(s), h$ is the maximum height, $\gamma$ represents the weight per unit length, and $\mathbf{k}$ is the unit vector in 
the $z$ direction, then the shape of the curve (represented by the position vector $\mathbf{r}(s))$ is found by solving the following system of differential equations derived from equations 1 and 2 :

$$
\theta^{\prime}(s)=\frac{\gamma \lambda}{T}(0.5 h-y(s)), r_{x}^{\prime}(s)=\lambda \cos \theta(s), r_{y}^{\prime}(s)=\lambda \sin \theta(s)
$$

where $\lambda$ represents the arc length and is equal to the magnitude of the derivative of position vector, $\mathbf{r}(s)$.

In the second approach, we employed a parametric deformable model in which a defined global deformation, with specific shape parameters, was applied to a geometric primitive [2]. The global deformation $(\mathbf{T})$ is defined as a lower pole, ptosis-like, deformation and is a function of the parameters $b_{0}$ and $b_{1}$. In the 2D case, using a half-circle, applying this deformation $(\mathbf{T})$ modifies the primitive such that the reference shape, s, can be defined as,

$$
\mathbf{s}=\mathbf{T}\left(\mathbf{e} ; \mathbf{b}_{\mathbf{0}}, \mathbf{b}_{\mathbf{1}}\right)=\left(\begin{array}{l}
r_{x} \\
r_{y}-b_{0} r_{x}-b_{1} r_{x}^{2}
\end{array}\right)
$$

where $\mathbf{e}$ is the geometric primitive with the $x$ axis in the anterior-posterior direction and the $y$ axis in the superior-inferior direction. As shown in the expression, the lower pole deformation affects the superior-inferior position of a point on the curve as a function of its anterior-posterior position. For the case of a half-circle, the shape equations are defined as,

$$
r_{x}=a_{1} \cos (\theta), r_{y}=a_{2} \sin (\theta), a_{1}=a_{2}=r_{0} .
$$

To relate the parameters $\mathbf{b}=\left[b_{0}, b_{1}\right]^{\top}$ for the lower pole deformation to realistic physical properties and predict the shape of the deformed curve, an optimization approach was used [6].

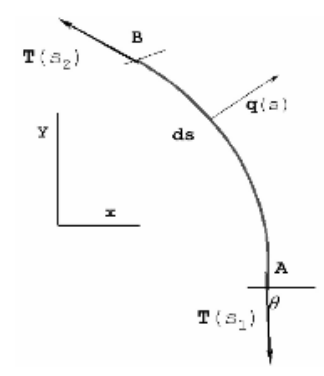

Fig. 1. Free body diagram of inextensible curve.

3D Modeling: Finite element (FE) analysis was used to perform 3D shape analysis by using the ABAQUS/Standard software [3]. The reconstructed breast was modeled using both idealized and realistic breast geometries that were created from geometric primitives and surface range data, respectively. Realistic breast geometries were created by scanning both phantoms made from silicone 
breast implants and human subjects. Scanning was performed using a Minolta Vivid $700{ }^{\circledR}$ digitizer system. The $3 \mathrm{D}$ scan point data were then converted to efficient CAD models for finite element analysis. Images of these shapes are shown in Figure 2,

Solid and shell elements were used to model homogeneous adipose tissue and skin, respectively. To represent the skin, finite membrane and shell elements were used. To model homogenous tissue, linear brick, hybrid elements were used. For the boundary condition, the posterior surfaces of the breast models were pinned to simulate the constraining of the breast to the chest wall and at the inframammary fold. The type of loading required depended on the type of reconstructive procedure modeled. For, example, hydrostatic pressure loads for implant reconstruction and uniform distributed surface pressure loads for TRAM reconstruction. Realistic mechanical tissue properties were incorporated using the Mooney-Rivlin strain energy density function based on published data for skin and soft tissue [45].

To gain an understanding of the initial and loading conditions necessary for the different types of reconstruction, the geometry of the undeformed shape of the breast was simplified as a half-sphere with a non-deformable posterior base, and the reconstructed breast treated as a homogenous, isotropic, incompressible body. The half-sphere was modeled as a hollow shell object. To represent forces that stretch the skin in implant reconstruction, a hydrostatic pressure load was applied normal to the skin membrane to denote the static fluid pressure in a saline-filled breast implant. To model TRAM-reconstruction, the half-sphere was modeled as a solid object, with an overlying skin membrane. Several approaches were used to model the skin-tissue interface such as: (a) modeling the breast as a composite object with a skin surface bonded to the tissue surface, and (b) defining an interaction property between the two surfaces and modeling the mechanical contact between them. To realistically model TRAM reconstruction, the surface interaction was defined since the skin is not directly bonded to the tissue. The mechanical contact between the skin and tissue surfaces was modeled with finite (i.e., small sliding) at the interface with the tissue surface defined as the master surface and the skin defined as a slave surface.

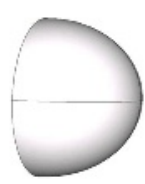

(a)

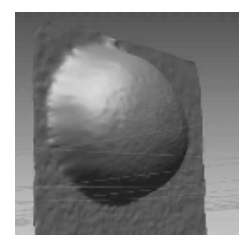

(b)

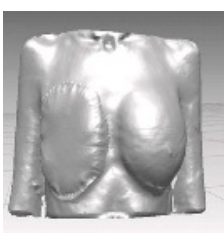

(c)

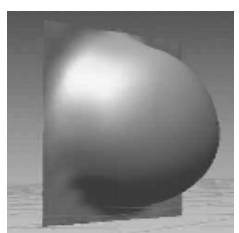

(d)

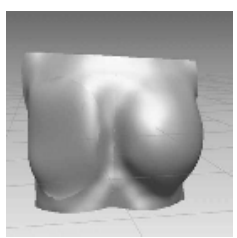

(e)

Fig. 2. Breast models: (a) Idealized breast shape of half-sphere. (b, c) Scanned data of implant and human torso. (d, e) Corresponding CAD/CAE surface models for FE analysis. 


\section{Results and Discussion}

2D Numerical Modeling (Approach One): Since $T$ (tension) and $\lambda$ are constants and not functions of $s$ anymore (as a consequence of the tangent equilibrium equation and the constitutive law), various shapes of the curve are found for a ratio of $\gamma$ to $T$ and different values of $\lambda$. Plots of the shape of the curves are shown in Figure 3. This initial 2D study showed that the tension is constant along the length of a curve (such as for an isotropic membrane) regardless of the material properties and the deformed shape is very dependent on the stretch material property, $\lambda$.

2D Numerical Modeling (Approach Two): By using a minimization technique to obtain the shape parameters and applying the deformation to an initial shape, the deformations, shown in Figure 4 were obtained. This simple forward 2D problem presented a good starting point for the shape analysis. However, tissue elasticity (stretch), skin anisotropy, and the three-dimensionality of the actual breast shape necessitated further study of the shape deformation problem in order to develop a predictive capability.

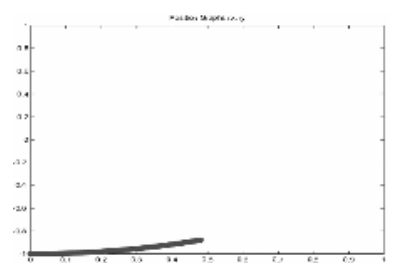

(a)

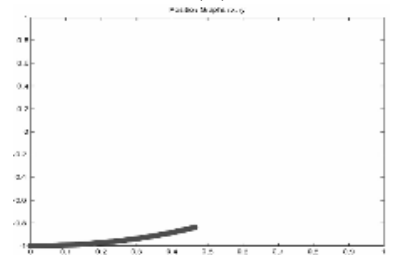

(d)

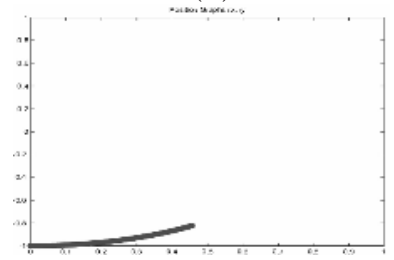

(g)

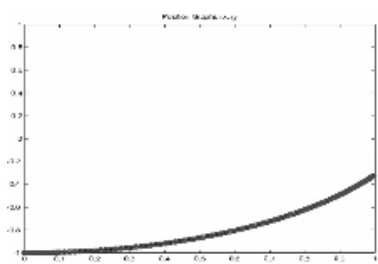

(b)

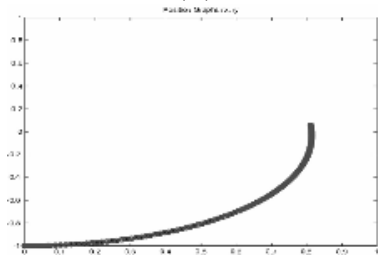

(e)

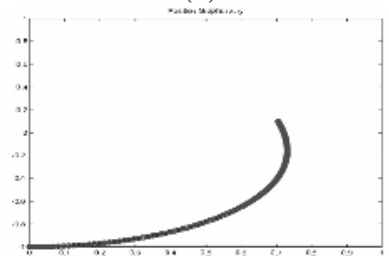

(h)

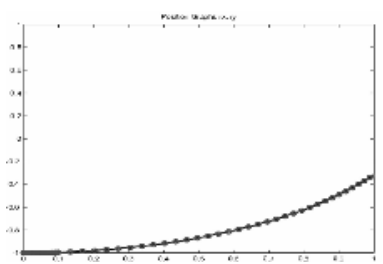

(c)

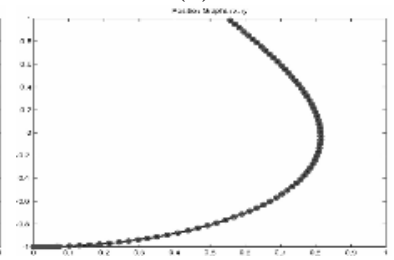

(f)

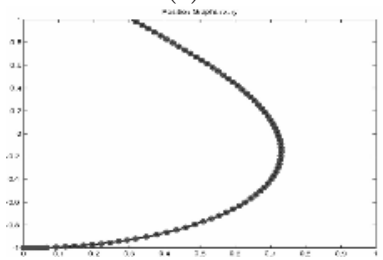

(i)

Fig. 3. Plots of $r_{x}$ versus $r_{y}$ as $\lambda$ varies from 0.5 to 3.5 (left to right) and the ratio $\gamma / T$ remains constant. (a-c) $\gamma / T=0.5$, (d-f) $\gamma / T=0.68$, (g-i) $\gamma / T=0.75$. 


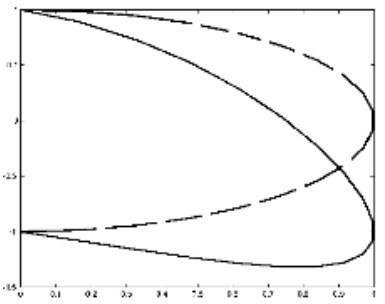

(a)

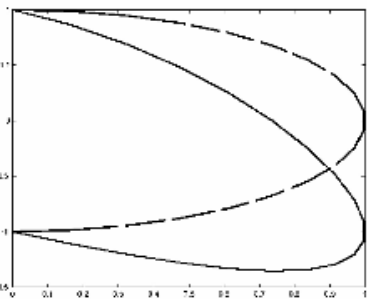

(b)

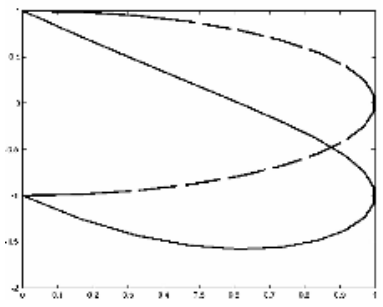

(c)

Fig. 4. Plots of lower pole deformation for various shape parameters. Solid line represents deformed shape. (a) $\mathrm{b}_{0}=0.5, \mathrm{~b}_{1}=0.5$; (b) $\mathrm{b}_{0}=0.6994, \mathrm{~b}_{1}=0.3006$; and (c) $\mathrm{b}_{0}=1.738$, $\mathrm{b}_{1}=-0.738$.

3D Modeling: As stated earlier, initially the breast shape was treated as a half-sphere elastic body with a uniform pressure load. Figure [5] depicts the deformation of the model in which the deformation of the breast shape depended on the pressure exerted on the inner boundary of the membrane and gravity. The initial results for the linear models indicate that the stresses at the posterior base of the solid object are significantly greater than the anterior stresses, as compared to the shell object. Also, while the displacement magnitude is greater for the shell object than for the solid object, the displacement distributions are similar.

Modeling of Implant-Based Reconstruction: The implant-based modeling, shown in Figures 6(a-b), simulated various volumes and modeled the stretching of skin due to the pressure exerted by the implant. For an $8 \mathrm{~cm}$ implant with a maximum volume of $150 \mathrm{cc}$, the pressure at the membrane is about $800 \mathrm{~Pa}$. This produces stresses up to $17 \mathrm{kPa}$ with $0.63 \mathrm{~cm}$ displacement. If we reduce the volume by half, the pressure becomes $400 \mathrm{~Pa}$ and stresses of $15 \mathrm{kPa}$ are produced. As shown in the images, as pressure increases, the stress in the medial-lateral regions of the lower half of the breast increases. Thus, as the volume increases, the medial-lateral stress will increase in a breast reconstructed with an implant.

Skin thickness and elasticity are important factors in obtaining favorable surgical outcomes. If the skin is too thin or inelastic it will not support an implant. Figures [6 (c-d) depict the deformation when the membrane is thinned from $1 \mathrm{~cm}$ to $0.25 \mathrm{~cm}$. The magnitude and area of the stress increases as thickness decreases. The stress increased from $17 \mathrm{kPa}$ to $24 \mathrm{kPa}$, with the medial-lateral stress increasing in both the upper and lower quadrants of the breast. These results indicate that as skin thickness decreases, stresses on the skin surrounding the implant tend to increase significantly.

Modeling of TRAM Reconstruction: In free TRAM reconstruction, the reconstructed tissue mound is tacked to the pectoralis major muscle and chest wall to hold it in place and the skin placed over the tissue. Figure 6)(e) depicts the deformation of the half-sphere modeled as a TRAM-based reconstructed breast. 
For the TRAM simulation, the stress is about $14.75 \mathrm{kPa}$ with maximum mediallateral and superior-inferior distributions, with large medial-lateral stress at the chest wall.

These results provided a reliable start for a computer simulation of the reconstructed breast where similar initial, boundary and loading conditions were applied to the realistic geometry models.

Simulation of Realistic Reconstructed Breast Models: Figures 7 (a-c) depict the deformation of a simulated implant reconstructed breast, in which a 300 cc implant, modeled as a hydrostatic pressure load, was applied to a skin membrane of $0.5 \mathrm{~cm}$ thickness. The resultant stress of about $4.5 \mathrm{MPa}$ is greatest in the medial-lateral regions. As shown, the deformed shape is not symmetrical to the opposite breast. This is due to several factors such as, insufficient initial

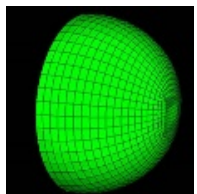

(a)

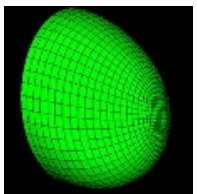

(b)

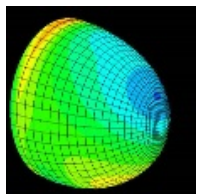

(c)

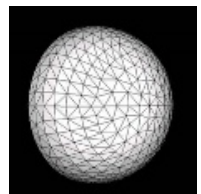

(d)

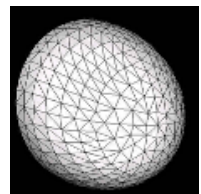

(e)

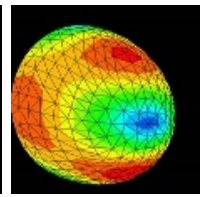

(f)

Fig. 5. Medial-lateral oblique views of deformation of (a) initial (undeformed) shape of shell, (b) shell object with inner pressure represented as a force due to a static fluid, (c) stress contour plot for the shell, (d) undeformed shape of solid, (e) solid object with a force due to tissue, and (f) stress contour plot for the solid.

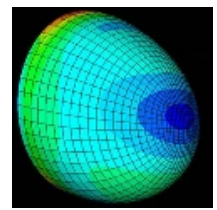

(a)

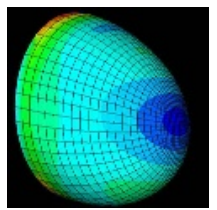

(b)

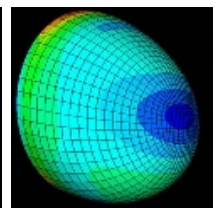

(c)

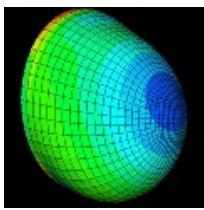

(d)

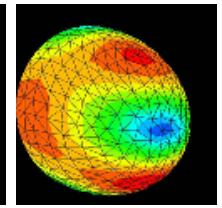

(e)

Fig. 6. Deformation of an implant reconstructed breast: (a) $800 \mathrm{~Pa}(175 \mathrm{cc}$ ) and (b) $400 \mathrm{~Pa}(87 \mathrm{cc})$. Deformation of an implant reconstructed breast with varying skin thickness: (c) $1 \mathrm{~cm}$ thickness and (d) $0.25 \mathrm{~cm}$ thickness. (e) Deformation of simulated TRAM reconstructed breast.

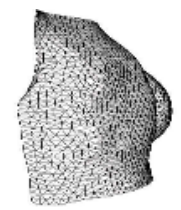

(a)

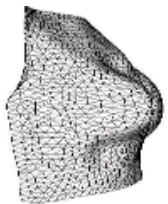

(b)

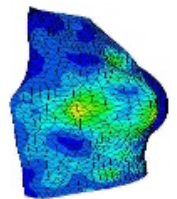

(c)

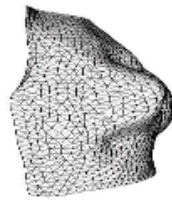

(d)

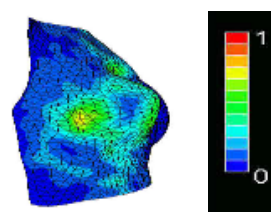

(e)

Fig. 7. (a) Initial shape. (b) Simulation of implant reconstructed breast (deformed shape). (c) Stress contour plot of (b). (d) Simulation of TRAM reconstructed breast (deformed shape). (e) Stress contour plot of (d). 
conditions and material properties. For instance, the undeformed structure is not an actual view of a site that has undergone mastectomy, but only a geometric representation and therefore the initial stress is unknown.

Recall that a TRAM reconstructed breast was modeled by simulating the stretching of the skin membrane by a hemispherical mound of tissue with the mechanical contact between the tissue and skin modeled as frictionless with finite sliding. The deformation is shown in Figures 7 (d-e). This simulation yields a maximum stress of $15 \mathrm{MPa}$. Observe that the deformed shape has less superior displacement than the implant-based simulated breast.

\section{Conclusion}

While further studies are warranted to determine appropriate material properties and initial conditions, development of these methods can lead to a range of clinically useful systems, such as surgical planning tools, physician training, and patient decision aids.

\section{References}

1. J. Bostwick. Plastic and Reconstructive Breast Surgery, volume 2. Quality Medical Publishing, Inc, St. Louis, 1990.

2. D. Chen, I.A. Kakadiaris, M. Miller, B. Loftin, and C. Patrick. Modeling for plastic and reconstructive breast surgery. In Medical Image Computing and Computer Assisted Intervention, pages 1040-1050, Pittsburgh, PA, October 2000. Medical Image Computing and Computer Assisted Intervention.

3. Hibbitt, Karlsson, and Sorensen. Abaqus Version 6.3. Hibbitt and Karsson and Sorensen, Inc., 1080 Main St Pawtucket, RI 02860, 6.3 edition, 2002.

4. Frederick Silver. Biological Materials:Structure, Mechanical Properties, and Modeling of Soft Tissues. New York University Press, 1987.

5. P. Wellman. Breast tissue stiffness in compression is correlated to histological diagnosis. Technical report, Harvard Bio-Robotics Lab, 1998.

6. C. Williams, I.A. Kakadiaris, K. Ravi-Chandar, M. Miller, and C. Patrick. Simulation studies for predicting surgical outcomes in breast reconstructive surgery. Technical report, Visual Computing Lab, Dept. of Computer Science, University of Houston, TX, 2003. 